\title{
Making sense of alcohol consumption data in Australia
}

\author{
Has alcohol consumption in Australia increased in recent years?
}

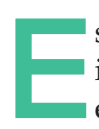

stimating the consumption of alcohol by

individuals and societies is notoriously difficult, especially as it frequently relies on self-reported data. Much depends on the quality of the datagathering instrument, the questions asked, and the veracity of the survey sample.

The Australian Bureau of Statistics (ABS) conducts periodic health surveys to collect information on the health of Australians, including data on their alcohol consumption. The 2001 National Health Survey ${ }^{1}$ was the first for which the ABS made the confidentialised unit record files available, enabling researchers to undertake detailed data analyses. Further health surveys were conducted in 2004-2005, 2007-2008 and most recently in 2011-2012. ${ }^{2}$ As is typical for ABS surveys, they were based on sophisticated sampling methods and face-to-face interviews conducted by specially trained staff, with response rates of $80 \%-90 \%$. These surveys are therefore of the highest quality. Alcohol consumption data were collected from respondents aged 18 years or over in the 2001 survey, and from those aged 15 years or older in the 2011-2012 survey. In this article, we compare the data from these two surveys to determine whether alcohol consumption in Australia changed over this period.

\section{Alcohol consumption by adults aged 18 years or over}

Respondents were asked when they had last had an alcoholic drink. In both surveys, just over $60 \%$ of adults had consumed alcohol during the past week, one in five reported that they had consumed alcohol in the past 12 months, just over $6 \%$ reported not having had a drink for more than 12 months, and $9 \%-10 \%$ claimed to have never consumed alcohol.

\section{"mean alcohol consumption for each} age group had increased between 2001 and 2017-2012"

\section{Sex- and age-related differences}

Respondents who reported having had an alcoholic drink during the week before the interview were asked to provide details about the amount, brand and type consumed during the most recent three days. This information was converted by the ABS

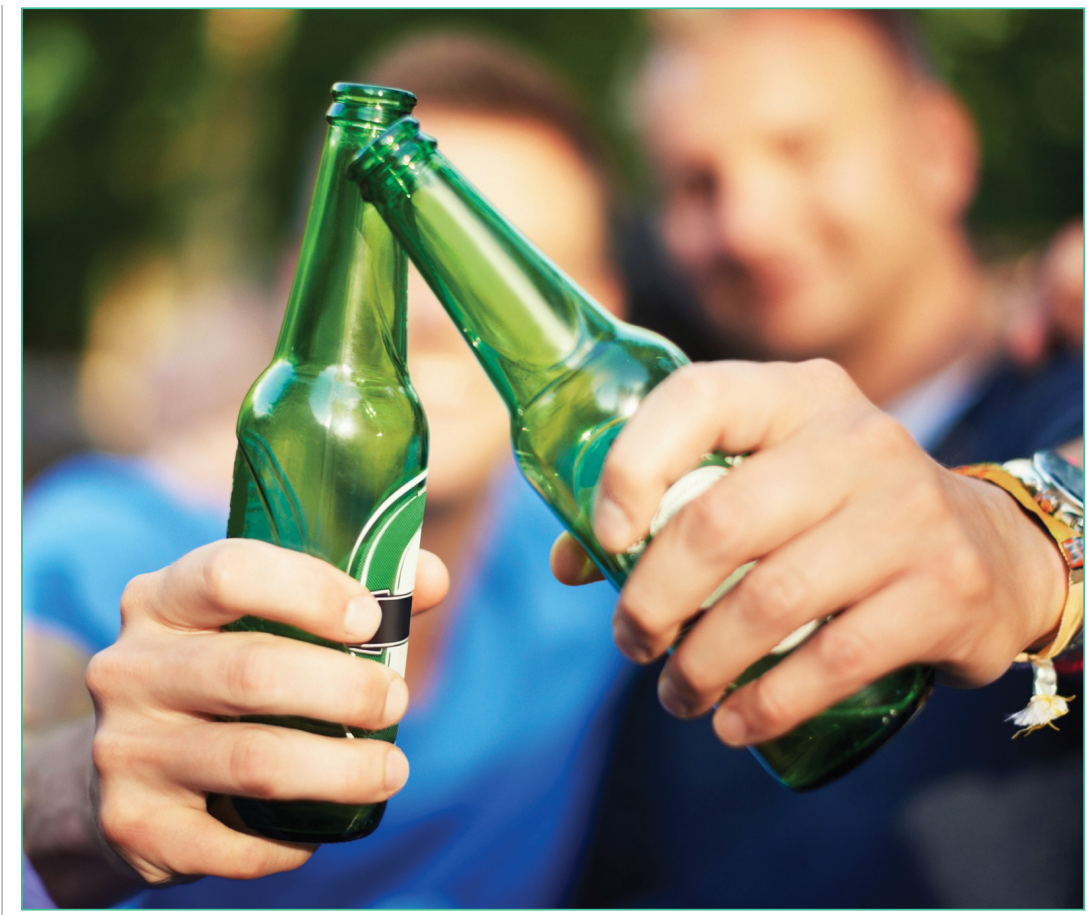

into numbers of standard drinks (equivalent to $10 \mathrm{~g}$ or $12.5 \mathrm{~mL}$ pure alcohol).

The weighted average daily alcohol intake increased between the two surveys by $13 \%$, from a mean ( \pm standard error) of $3.9 \pm 0.05$ in 2001 to $4.3 \pm 0.06$ standard drinks in 2011-2012. Over the same period, consumption among men increased from $4.7 \pm 0.07$ to $5.0 \pm 0.10$ standard drinks, and for women from $2.8 \pm 0.06$ to $3.4 \pm 0.07$ standard drinks. While women consumed much less alcohol than men in each survey, the gender gap had narrowed - women had consumed $40 \%$ less alcohol than men in 2001, but only 33\% less during 2011-2012.

Farhat Yusuf BSc(Hons), MA, PhD 1.2

Stephen R Leeder $M D, P h D, F R A C P^{\prime}$

1 Menzies Centre for Health Policy, University of Sydney, Sydney, NSW. 2 Department of Marketing and Management, Macquarie University, Sydney, NSW.

farhat.yusuf@ sydney.edu.au

doi: 10.5694/mjal5.00151
Analysis of the alcohol consumption data by age group identified three important features:

- alcohol consumption tended to decline with increasing age;

- mean alcohol consumption for each age group had increased between 2001 and 2011-2012;

- the increase was statistically significant only for those under 55 years of age (Box 1).

Disaggregating the age-specific consumption data by sex revealed similar patterns. 
1 Weighted average daily alcohol intake over days recorded (with standard errors) by age

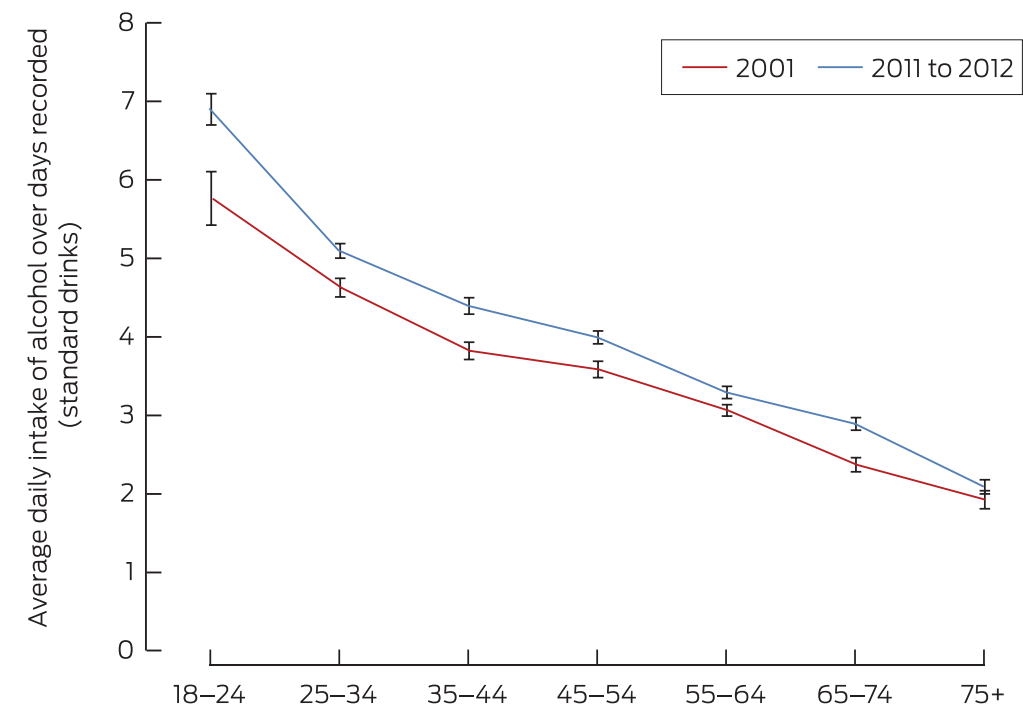

Source: National Health Survey, 2001 and Australian Health Survey, 2011-2012.

\section{Alcohol consumption by teenagers aged 15-19 years}

As noted earlier, information on alcohol consumption among teenagers aged 15-19 years was collected during the 2011-2012 health survey, but not in the 2001 health survey. In this age group, $41.6 \% \pm 2.89 \%$ had consumed alcohol during the week before the interview, compared with $60.1 \% \pm 0.61 \%$ of those 18 years or older. On the other hand, $21.1 \% \pm 1.67 \%$ of those aged $15-19$ years but only $9.0 \% \pm 0.36 \%$ of those 18 years or older reported having never consumed alcohol.

The average daily consumption over days recorded for those aged 15-19 years was $7.1 \pm 0.45$ standard drinks. This was $65 \%$ higher than the average for adults (4.3 \pm 0.06 standard drinks). The gender gap in alcohol consumption for this age group was only $9 \%$, compared with $33 \%$ for adults.

\section{The socioeconomic gradient in alcohol consumption}

Analysis of the data from both surveys in terms of the education, occupation and labour force status of respondents indicated that tertiary qualifications, employment and white-collar occupations were associated with lower daily levels of alcohol consumption.

The socioeconomic gradient in alcohol consumption was also observed when data from the 2011-2012 survey were analysed according to the Index of Relative Socio-economic Disadvantage (IRSD) for each respondent's neighbourhood. The IRSD is one of the multidimensional Socio-Economic Indexes for Areas (SEIFA) calculated by the ABS from 2011 census data on variables such as education, occupation, unemployment and household income. ${ }^{3}$

Box 2 presents the weighted average daily alcohol intake over days recorded by IRSD quintiles. Quintile 1 includes areas with the highest levels of socioeconomic disadvantage (ie, the most disadvantaged 20\%) and quintile 5 the least disadvantaged. The difference in alcohol consumption between the highest and lowest quintiles was in the order of $21 \%$ for adults (aged 18 years or older) and 34\% for teenagers (15-19 years). Mean alcohol consumption among teenagers was substantially higher than for adults in every quintile.

Household expenditure on alcohol is another aspect of alcohol consumption relevant to health: money spent on alcohol cannot be used to buy food. Using data from the Household Expenditure Survey (HES) of 2003-2004, Yusuf and de Meyrick ${ }^{4}$ found that the average weekly expenditure on alcohol by households that reported purchasing it was \$39.95 \pm \$0.91, equivalent to $3.8 \%$ of total household expenditure on all goods and services. Similar analysis of the 2009-2010 HES data indicated that the average weekly expenditure on alcohol had increased to $\$ 56.50 \pm \$ 1.50$, but as a proportion of total household expenditure it had only increased to $3.9 \%$, which was not a significant change from the previous survey.

Disaggregating the expenditure data from the 20092010 HES according to household income quintile showed that households in the lowest quintile spent

2 Weighted average daily alcohol intake of over days recorded (with standard errors) by Index of Relative Socio-Economic Disadvantage quintiles for adults (over 18 years of age) and teenagers (15-19 years of age)

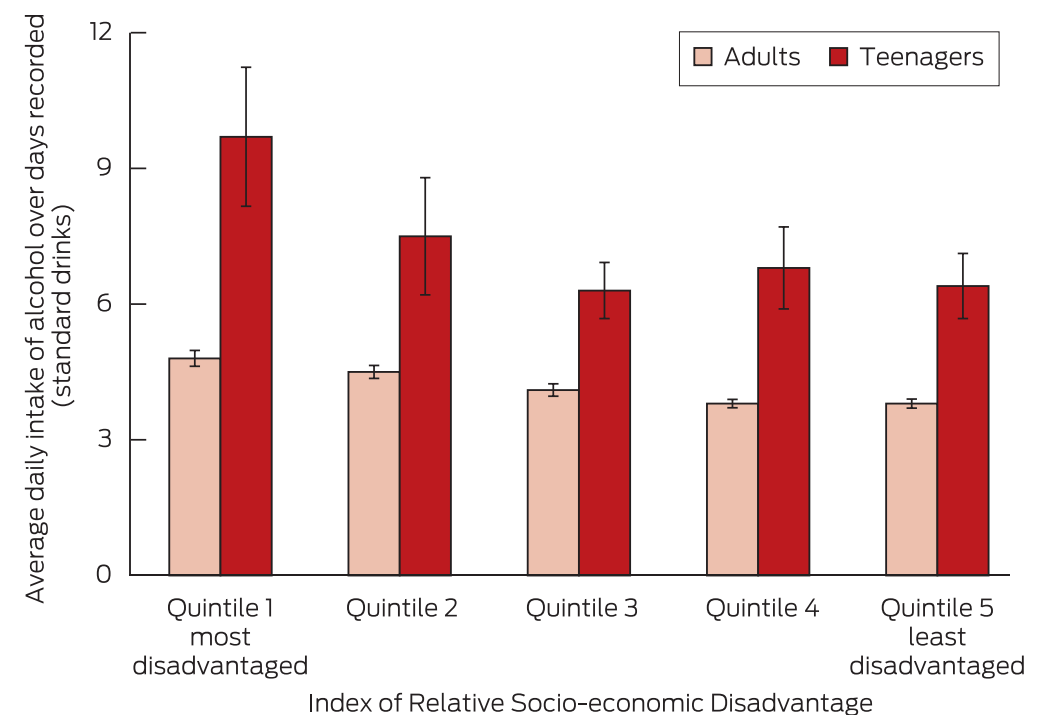

Source: Australian Health Survey, 2011-2012. 
$\$ 31.34 \pm \$ 1.58$ per week on alcohol, while those in the highest income quintile spent $\$ 381.68 \pm \$ 4.15$. But the poorer households spent a much greater proportion of their total household budget on alcohol than their wealthier counterparts: $4.6 \%$ compared with $3.6 \%$.

\section{NHMRC guidelines and risky levels of alcohol consumption}

Both health surveys also analysed alcohol consumption data in terms of the 2001 National Health and Medical Research Council (NHMRC) guidelines for minimising the longer-term risk posed by alcohol consumption (low-risk intake for men: up to 4 standard drinks per day, medium risk: 5-6, and high risk: more than 7 standard drinks per day; for women: about half these levels). ${ }^{5}$

While the sex differences in alcohol consumption we noted earlier persisted, $50.9 \% \pm 0.51 \%$ of all respondents aged 18 years or over who reported drinking during the week before the 2001 survey were in the low-risk category, $6.7 \% \pm 0.24 \%$ in the mediumrisk category and $4.1 \% \pm 0.19 \%$ in the high-risk category. The corresponding statistics for the 2011-2012 survey were $48.0 \% \pm 0.55 \%, 7.1 \% \pm 0.26 \%$ and $4.7 \% \pm$ $0.24 \%$, respectively. These figures indicate a decline between the two surveys in the proportion drinking at the low-risk level, with increases in both the mediumand high-risk categories. A comparison for drinkers younger than 18 years was not possible because of the unavailability of data in the 2001 health survey.

The 2009 NHMRC guidelines for reducing lifetime risk from drinking alcohol ${ }^{6}$ were used to analyse the 2011-2012 survey. Unlike the 2001 NHMRC guidelines, the 2009 guidelines do not provide separate recommendations for men and women. In order to reduce the lifetime risk of disease or injury, they recommend "drinking no more than two standard drinks on any day" and "no more than four standard drinks on a single occasion".

The number of standard drinks consumed during the week before the interview (7-day average) was used to identify respondents who exceeded the 2009 NHMRC guidelines. Among those 18 years of age or older, $29.2 \% \pm 0.73 \%$ of men and $10.1 \% \pm 0.51 \%$ of women had exceeded the 2009 guidelines' safe level, while the corresponding figures for those aged 15-19 years were $35.0 \% \pm 4.57 \%$ (men) and $19.8 \% \pm 3.57 \%$ (women). Box 3 depicts the percentages of persons exceeding the 2009 guidelines by age and sex. For every age group, the proportion of men exceeding the guidelines was significantly greater than that of women, consistent with the sex differences in alcohol consumption noted earlier.

\section{Conclusion}

Our analysis of the data from two nationally representative surveys suggests that the consumption of alcohol in Australia has increased in recent years. A sex difference in alcohol consumption by adults was evident in both surveys. Based on the 2011-2012 survey, the prevalence of alcohol intake among those aged 15-19 years was significantly higher than in adults. Moreover, relatively disadvantaged people reported higher consumption levels, and they also spent a somewhat larger proportion of their household budget on alcohol.

Our finding of a trend to increased alcohol consumption contrasts with that of an Australian Institute of Health and Welfare (AIHW) survey ${ }^{7}$ which found a decline in alcohol consumption in recent years. This may be due to methodological differences between the ABS and AIHW surveys. One major difference was the reference period for collecting alcohol consumption data: one week before the interview in ABS surveys, but one year before the interview in the AIHW surveys. Another was the much lower response rate in the AIHW surveys about half of that for the ABS surveys.

However, our findings regarding the sex differences, age patterns and the socioeconomic gradient in alcohol consumption were similar to those reported by the AIHW. A socioeconomic gradient in levels of alcohol consumption was also noted in a study conducted in the United Kingdom. ${ }^{8}$

Competing interests: No relevant disclosures.

Provenance: Not commissioned; externally peer reviewed.

(c) 2015 AMPCo Pty Ltd. Produced with Elsevier B.V. All rights reserved.

References are available online at www.mja.com.au.

3 The proportions of respondents whose alcohol consumption (7-day average) exceeded the 2009 National Health and Medical Research Council recommendations for safe drinking during the week before the interview, by age and sex

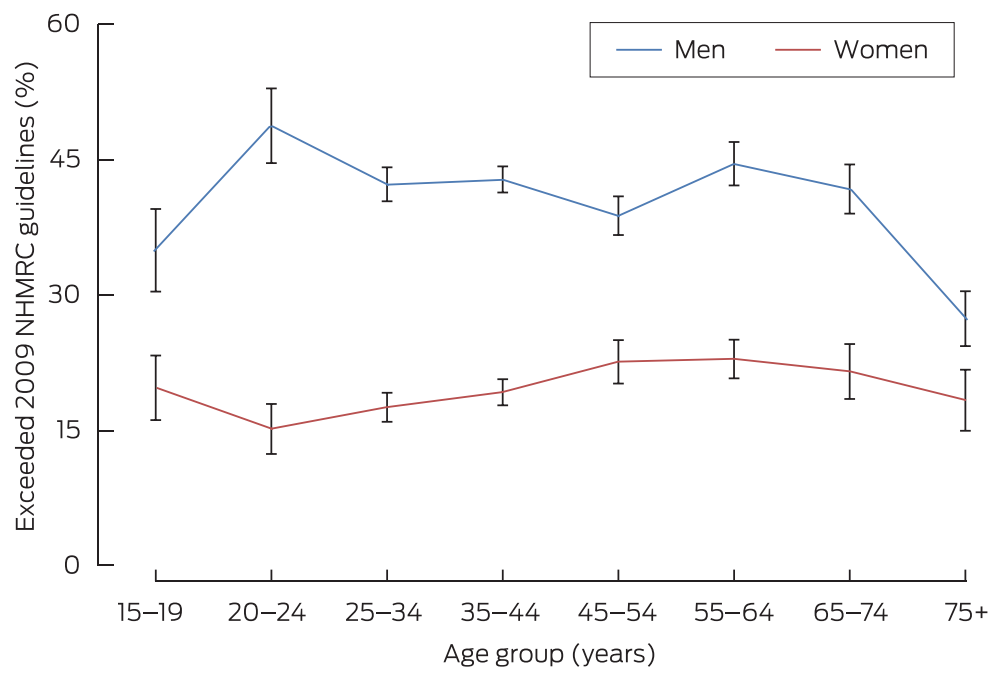

Source: Australian Health Survey, 2011-2012. 
1 Australian Bureau of Statistics. National Health Survey: users' guide, 2001. Canberra: ABS, 2003. (ABS Cat. No. 4363.0.55.001.) http://www.abs.gov.au/AUSSTATS/abs@.nsf/66f306f503e52 9a5ca25697e001766lf/11b070b0d4af5baeca256d32001b04b 4!OpenDocument (accessed Oct 2014).

2 Australian Bureau of Statistics. Australian Health Survey: users' guide, 2011-13. Canberra: ABS, 2013. (ABS Cat. No. 4363.0.55.001.) http://www.abs.gov.au/AUSSTATS/abs@.nsf/ Lookup/4363.0.55.001Main+Features12011-13?OpenDocument (accessed Nov 2014).

3 Australian Bureau of Statistics. Census of population and housing: Socio-Economic Indexes for Areas (SEIFA), Australia, 2011. Canberra: ABS, 2013. (ABS Cat. No. 2033.0.55.001.) http:// www.abs.gov.au/ausstats/abs@.nsf/mf/2033.0.55.001 (accessed Nov 2014).

4 Yusuf F, de Meyrick J. Alcohol buying behaviour of Australian households. In: Hoque N, Swanson DA, editors. Opportunities and challenges for applied demography in the 21st century. Dordrecht: Springer, 2012: 175-194.

5 National Health and Medical Research Council. Australian alcohol guidelines: health risks and benefits. Canberra: NHMRC, 2001. http://www.nhmrc.gov.au/guidelines/ publications/ds9 (accessed Nov 2014).

6 National Health and Medical Research Council. Australian guidelines to reduce health risks from drinking alcohol. Canberra: NHMRC, 2009. http://www.nhmrc.gov.au/ guidelines/publications/ds10 (accessed Nov 2014).

7 Australian Institute of Health and Welfare. National Drug Strategy Household Survey detailed report: 2013. Canberra: AlHW, 2014. http://www.aihw.gov.au/publicationdetail/?id=60129549469 (accessed Nov 2014).

8 Fone D, Farewell D, White J, et al. Socioeconomic patterning of excess alcohol consumption and binge drinking: a crosssectional study of multilevel associations with neighbourhood deprivation. BMJ Open 2013; 3: e002337. 